American Journal of Applied Sciences 5 (11): 1499-1505, 2008

ISSN 1546-9239

(C) 2008 Science Publications

\title{
A New Method for Optimal Harmonic Meter Placement
}

\author{
A. Ketabi and S.A. Hosseini \\ Department of Electrical Engineering, University of Kashan, Kashan, Iran
}

\begin{abstract}
In this research, a new method based on Singular Value Decomposition (SVD) is proposed to solve the problem of optimal placement of meters for static estimation of harmonic sources in a power system. Also, the Binary Genetic Algorithm (BGA) is used to solve the problem of optimization. IEEE 14-bus test system is provided to validate the measurement placement algorithm. It has been observed that the quality of estimation is improved and the Number of Observable Variable (NOV) is increased. Moreover, the BGA-based meter placement strategy yields the same solution as obtained from the complete enumeration technique but in shorter time.
\end{abstract}

Key words: Harmonic state estimation (HSE), singular value decomposition, optimal meter placement, binary Genetic algorithm (BGA)

\section{INTRODUCTION}

Harmonic distortion has experienced a continuous increase in power systems owing to the growing use of nonlinear loads. Many studies have shown that harmonics may cause serious effects on power systems, communication systems and various apparatus ${ }^{[1]}$. As a result, there is a growing concern to limit the amount of harmonics in the system and out of this concern, harmonic standards have been formulated ${ }^{[2]}$. To effectively evaluate and diminish the harmonic distortion in power systems, the locations and magnitudes of harmonic sources have to be identified. This problem of determination of locations and magnitudes of the harmonic sources is generally termed as "reverse harmonic power flow problem ${ }^{[3]}$ and to solve it, appropriate locations of the harmonic meters are very important ${ }^{[7]}$. The number of harmonic instruments available is always limited due to cost and the quality of the estimates is a function of the number and location of the measurement points ${ }^{[7]}$. Therefore, a systematic procedure is needed to design the optimal measurement placement.

A measurement placement algorithm for harmonic component identification is presented in $^{[4]}$, based on minimum variance criteria. The optimal procedure $\mathrm{in}^{[4]}$ needs load and generation data at each harmonic order for all busbars, which is usually not available. $\operatorname{In}^{[5]}$ a symbolic method for observability analysis is presented. This method identifies redundant measurements thus giving the minimum number of measurements that are needed to perform HSE. It should be noted that this method cannot detect cases when there are two dependent measurement equations because the actual values are lost. Also $\mathrm{In}^{[6]}$ a technique based on the minimum Condition Number $(\mathrm{CN})$ of the measurement matrix has been proposed for designing the harmonic instruments points. As shown in this research, minimizing the $\mathrm{CN}$ of the measurement matrix doesn't necessarily lead to the better estimation quality. Thus, an alternative criteria for deciding the optimal measuring locations is still needed.

The optimization technique used to solve the problem is also an important issue which needs to be determined with care. Farach and Grady ${ }^{[4]}$ have developed a Sequential Procedure (SP) for this object. In this method, for an N-bus system with $\mathrm{P}$ meters, only $\mathrm{P}(2 \mathrm{~N}+1-\mathrm{P}) / 2$ combinations of meter placement need to be checked. Moreover, the SP yields only a good approximate solution and the best solution can only be obtained by Complete Enumeration (CE) of all the combinations of meter placements ${ }^{[7]}$. But CE technique for finding optimal meter placement is too exhaustive even for a moderate size of power system and hence cannot be implemented practically ${ }^{[7]}$. Thus an alternative method for deciding the optimal measuring locations, which gives the same locations as that obtained by the CE technique, is still needed. $\operatorname{In}^{[7]} \mathrm{a}$ genetic algorithm-based method has been proposed to solve the problem of optimal meter placement. It is worth noting that line measurements have not been used in $^{[7]}$ and the optimization problem has been solved with Continuous Genetic Algorithm (CGA).

Corresponding Author: A. Ketabi, Department of Electrical Engineering, University of Kashan, Kashan, Iran Tel/Fax: +98-3615559930 
To address the above mentioned needs, in present research, a genetic algorithm-based method has been proposed to solve the problem of optimal meter placement for HSE. For fitness evaluation of the GA strings, a new criteria based on SVD has been presented in this work. It has been found that using the obtained combination of meters from the proposed criteria, the quality of the estimation is improved and, also GAbased technique gives the same results as that obtained by the $\mathrm{CE}$ technique and is better than the sequential technique.

\section{HARMONIC STATE ESTIMATION}

Harmonic state estimation technique uses few synchronized harmonic measurement data as input to find the complete harmonic information for the whole network ${ }^{[8-10]}$. A framework of HSE can be found in ${ }^{[11]}$.

A general mathematical model which relates the measurement vector $\mathrm{Z}$ to the state variable vector $\mathrm{X}$ which is to be estimated can be formulated as follows:

$$
Z(h)=H(h) X(h)+E(h)
$$

where, $\mathrm{Z}(\mathrm{h})$ is a measurements vector, $\mathrm{H}(\mathrm{h})$ is a measurement matrix, $\mathrm{X}(\mathrm{h})$ is a state vector to be estimated, $\mathrm{E}(\mathrm{h})$ is the measurement noise at $\mathrm{h}^{\text {th }}$ harmonic order.

The measurement matrix can be considered as the matrix whose elements relate the measurement vector to the state variable vector. As a result, the proposed algorithm considers only one harmonic order of at a time and the variable of $\mathrm{h}^{\text {th }}$ harmonic order in the previous equation will be left.

Various methods are possible, the most widely used is the Weighted Least Square (WLS). The WLS estimate is, therefore, the vector $\mathbf{X}$ that minimizes the weighted sum of the squares of the residuals $(\mathrm{R}=\mathrm{Z}-\mathrm{HX})$ between the actual measurements and estimates levels, i.e.,

$$
\text { Minimise } J(X)=(Z-H X)^{T} R^{-1}(Z-H X)
$$

where, $\mathrm{R}^{-1}$ is the inverse of the covariance matrix.

Matrix $\mathrm{R}$ is diagonal and contains the covariances of the measurements (if they are known). This permits applying higher weighting to measurements that are known to be more accurate. $\mathrm{R}$ is replaced by the identity matrix if the same instrumentation is used to obtain them ${ }^{[14]}$.

The solution to (1) in the WLS sense is obtained by solving the following equation:

$$
\left(H^{T} R^{-1} H\right) X=\left(H^{T} R^{-1}\right) Z
$$

The measurement equation can be linear by choosing the phasor busbar voltages as state variables and measuring phasor busbar voltages, phasor line and injection currents ${ }^{[14]}$. It is important to emphasize that the estimation algorithm for this case is direct (not iterative).

Equation 1 is usually under-determined system because of limitation of harmonic instruments and different ownership of different parts of the system. This results in $\left(\mathrm{H}^{\mathrm{T}} \mathrm{R}^{-1} \mathrm{H}\right)$ being singular and a result can not be obtained with normal equation approach ${ }^{[11]}$. Furthermore, even in completely or ever-determined system, the normal equations may be very singular or ill-conditioned. Although several methods have been suggested to solve such ill-conditioned problem, e.g., ${ }^{[12,13]}$, observability analysis is still needed prior to estimation.

\section{SINGULAR VALUE DECOMPOSITION (SVD)}

Principles of the SVD: To solve the HSE problem when only observable islands exist, SVD needs to be applied, since standard techniques for solving such equations will fail ${ }^{[11]}$. In addition using SVD removes the need for observability ${ }^{[5]}$.

The matrix $H(m \times n)$ of $(1)$ can be replaced using the SVD by product of three matrices, i.e.,

$$
\mathrm{H}=\mathrm{UWW}^{\mathrm{T}}
$$

where, $\mathrm{W}$ is a diagonal matrix $(\mathrm{n} \times \mathrm{n})$ with positive or zero elements, which are the singular values of $H$. $U$ is a column orthogonal $(\mathrm{m} \times \mathrm{n})$ matrix and $\mathrm{V}^{\mathrm{T}}$ is the transpose of an $(n \times n)$ orthogonal matrix.

SVD constructs special orthonormal bases for the null space and range of a matrix. It can be shown that $U$ is eigenvector matrix of $\mathrm{HH}^{\mathrm{T}}$ and $\mathrm{V}$ is the eigenvector matrix of $\mathrm{H}^{\mathrm{T}} \mathrm{H}$. Moreover, $\mathrm{WW}^{\mathrm{T}}$ is a diagonal matrix of eigenvalues. The column of $\mathrm{U}$, corresponding to the nonzero singular values are an orthonormal set of basis vectors that span over the range of $H$. The column of $\mathrm{V}$, corresponding to the zero singular values are an orthonormal set of basis vectors that span over the null space $^{[6]}$.

The solution process for measurement systems using SVD can be found in ${ }^{[11]}$. Equation (1) is solved in the WLS sense for over-determined measurement system as: 


$$
\mathrm{X}=\left(\mathrm{H}^{\mathrm{T}} \mathrm{H}\right)^{-1} \mathrm{H}^{\mathrm{T}} \mathrm{Z}
$$

Substitute SVD of gain matrix into (5), it yields:

$$
X=\left(V W^{T} U^{T} U W V^{T}\right)^{-1} V W^{T} U^{T} Z=V W^{-1} U^{T} Z
$$

Also for completely determined and underdetermined systems, the Eq. 6 is valid.

Detection unobservable region using SVD: In singular measurement systems, while most traditional techniques fail, SVD is able to provide a particular solution and a null space vector for each singularity. In such cases, there is no unique solution but an infinite number of solutions. The infinite solutions of such system are expressed as ${ }^{[5]}$ :

$$
[\mathrm{x}]=\left[\mathrm{x}_{\mathrm{P}}\right]+\sum_{\mathrm{i}=1}^{\mathrm{n}-\mathrm{rank}(\mathrm{H})} \mathrm{k}_{\mathrm{i}}\left[\mathrm{x}_{\mathrm{ni}}\right]
$$

where, $\left[\mathrm{x}_{\mathrm{P}}\right]$ is the particular solution, $\mathrm{k}_{\mathrm{i}}$ is a constant and $\left[\mathrm{x}_{\mathrm{ni}}\right]$ is the null space vector.

The null space vectors can be multiplied by any constant and added to the particular solution to give another valid solution to the set of equations, thereby specifying the infinite number of solutions. Variables corresponding to zeros in all the null space vectors will not be changed by this process and, hence, are completely specified by the particular solution. These variables correspond to estimates of quantities in the observable islands. The variables corresponding to nonzero elements in the null space vectors are in the unobservable regions as they cannot be uniquely determined $^{[5]}$.

\section{OPTIMAL HARMONIC METER PLACEMENT}

However, the number of measuring devices available is limited due to cost and the quality of estimates is a function of the number and locations of the measurements. A proper methodology is needed for selecting optimal sites for the measuring devices. In this section a new criteria is proposed for optimal harmonic meter placement. Since the proposed method is based on the SVD and minimum condition number of the measurement matrix, the minimum condition number criteria is first expressed for the sake of clarity followed by the proposed criteria.
Minimum condition number criteria: In solving linear equations, condition number relates the results variations (x) to the data variations (D):

$$
\frac{\|\overline{\mathrm{x}}-\mathrm{x}\|}{\|\mathrm{x}\|}=\mathrm{CN} \frac{\|\overline{\mathrm{D}}-\mathrm{D}\|}{\|\mathrm{D}\|}
$$

Based on the above definition, $\mathrm{CN}$ shows sensitivity of mathematical equation to the variations of data. For example consider the system $\mathrm{Ax}=\mathrm{b}$ with following matrixes:

$$
\mathrm{A}=\left[\begin{array}{cc}
0.78 & 0.563 \\
0.913 & 0.659
\end{array}\right], \quad \mathrm{b}=\left[\begin{array}{l}
0.217 \\
0.254
\end{array}\right]
$$

True answer of this equation is:

$$
\mathrm{x}=\left[\begin{array}{c}
1 \\
-1
\end{array}\right]
$$

If there are some errors in the equation data (A) as:

$$
E=\left[\begin{array}{cc}
0.001 & 0.001 \\
-0.002 & -0.001
\end{array}\right]
$$

Then after solving the equation $(\mathrm{A}+\mathrm{E}) \mathrm{x}=\mathrm{b}$, the answer will be:

$$
\left[\begin{array}{c}
-5 \\
-7.3085
\end{array}\right]
$$

This shows that a small variation of data leads to great variations of response. Now the $\mathrm{CN}$ of matrix (A) is calculated:

$$
\begin{aligned}
& \frac{\|\Delta \mathbf{A}\|_{2}}{\|\mathbf{A}\|_{2}} \approx 0.0018, \frac{\|\Delta \mathbf{x}\|_{2}}{\|\mathbf{x}\|_{2}} \approx 6.1562, \\
& \mathrm{CN}=\frac{6.1562}{0.0018}=3420.1
\end{aligned}
$$

It's observed that the $\mathrm{CN}$ of matrix (A) is too large.

As shown $\mathrm{in}^{[3]}$ the $\mathrm{CN}$ of a matrix is the ratio of the largest (in magnitude) to the smallest singular value. A matrix is singular if its condition number is infinite and it would be considered ill-conditioned if its condition number is too large.

Based on this criteria, combination that the $\mathrm{CN}$ of its measurement matrix is a minimum is selected as optimal combination for available meters. 
Proposed criteria: As stated in section III, if all the null space vectors have zero entries in a particular position, the corresponding state variable will be observable as any linear combination of null space vectors will not alter its value and can be estimated without any error. Based on this subject, a new algorithm is proposed for placing available meters:

Step 1: First, combination is searched for meters with entire nonzero singular value elements. If various combinations are found with this property, combination with the least $\mathrm{CN}$ of measurement matrix is selected. Otherwise, the next step is used.

Step 2: If combination for measurements with entire nonzero singular value elements not found, combination that null space vectors of its measurement matrix have more zero entries is selected. In fact in this method more variables are estimated without error (the Number of Observable Variables (NOV) will be increased).

Step 3: If several combinations are found with the same $\mathrm{NOV}$, combination with the least $\mathrm{CN}$ of measurement matrix is selected.

However, to improve the quality of state estimation, virtual and pseudo measurements can be included in the measurement matrix. Virtual measurements provide the kind of information that does not need metering (e.g., zero harmonic current injections at switching substation and at nonharmonic source bus ${ }^{[6]}$.

\section{BGA-BASED METE PLACEMENT}

Genetic algorithm searches for an optimal solution using the principles of evolution and heredity. By simulating the survival of the fittest evolution strategy among chromosome (i.e., string) structures, the optimal string is searched by randomized information exchange. The major advantage of using the GA is that the solution obtained is globally optimal ${ }^{[7]}$. Also GA is capable of obtaining the global solution of a wide variety of functions such as differentiable or nondifferentiable, linear or nonlinear, continuous or discrete and analytical or procedural ${ }^{[16]}$. For the problem of optimal meter placement for static harmonic estimation, the global solution obtained using GA requires a lesser number of iterations compared to the $\mathrm{CE}$ technique and at the same time eliminates the disadvantage of the sequential meter placement technique, which gives suboptimal solution ${ }^{[7]}$.
The placement of measurement points is normally assumed to be symmetrical (e.g., either three or no phases measured at a location). All possible measurement locations for an N-bus system in this research include all injection currents ( $\mathrm{N}$ locations), all node voltages ( $\mathrm{N}$ locations) and all line currents ( $\mathrm{L}$ locations, both sending and receiving ends). Therefore all possible measuring locations are equal $2 \mathrm{~N}+\mathrm{L}$. The algorithm of the proposed BGA technique is described in detail as follows.

The BGA begins, by defining the optimization variables and population size in each generation. The size of each string is considered to be a row vector with ' $2 \mathrm{~N}+\mathrm{L}$ ' elements, which have been combined zero and one values. In each string elements one and zero imply existing and not existing measurement device in related location respectively. Each string is actually denoting some combination of the meter locations and the number of each chromosome's ones is equal to the number of available harmonic meters (m). Hence, each element of each string should be initialized to represent a particular location, where a meter to be placed. In the present work, the population size (i.e., the number of strings) has been taken to be 10 .

The proposed criteria is to find combination for meters so that the number of observable variables (NOV) is maximized and the $\mathrm{CN}$ of measurement matrix is minimized (approach to one). Therefore the cost function is selected as below:

$$
\text { Cost Function }=\mathrm{CN}+\mathrm{k} \frac{1}{\mathrm{NOV}}
$$

where, coefficient $\mathrm{k}$ is selected so that the influence of $\mathrm{CN}$ and NOV be reasonable in the cost function.

To produce the new generation, the amount of $X_{\text {rate, }}$, the percent of chromosomes that are survived and directly transferred to the next generation, has been selected equal to $50 \%$. For the selection of parents' chromosomes, the tournament selection method is used. The best chromosome (the first) becomes a parent and the other parent is selected from the remaining of the $50 \%$ of the first chromosomes. This operation is repeated until all of the needed offspring are produced.

In this step, the next generation of GA is created as described below. The two strings, whose fitness values are the lowest and the second lowest respectively, are directly copied to the next generation. Then, by performing crossover another strings for the next generation are created as following. Two numbers are randomly created between the first and last bits of the parents' chromosomes that are termed as "crossover points". The bits between these two numbers are 
exchanged between offspring cruciformly and the bits that are outside of crossover points are directly transferred to the related offspring. The number of each chromosome's ones is equal to the number of available harmonic meters. Therefore the number of offspring' ones is checked and if it is less than ' $\mathrm{m}$ ', chromosome's zeros is randomly selected and converted to one; if it is more than ' $\mathrm{m}$ ', chromosome's ones is randomly selected and converted to zero.

If the best string does not change for two successive generations, then the mutation operator is applied to it. Over the first chromosome, which has the least amount of costs, mutation won't be done and is directly transferred to the next generation. After selecting the location of bits which mutation is done; if these bits are zero converted to one and if they are one converted to zero. However, it's noted that in this work the amount of $\mu$, the percent of population bits that mutation is done over them, is selected equal to $20 \%$.

After mating, mutation and ranking, the population is used as the starting population for the next generation. The number of generations that evolve depends on whether an acceptable solution is reached or a set number of iterations is exceeded. After a while all the chromosomes and associated costs would become the same if it were not for mutations, at this point the algorithm should be stopped ${ }^{[16]}$.

Figure 1 shows the flowchart of optimal measurement placement algorithm.

\section{TEST SYSTEM AND SIMULATION RESULTS}

In this section IEEE 14-bus test system is used to test the proposed measurement placement algorithm. A schematic of this test system is shown in Fig. 2 and its total data are provided from ${ }^{[15]}$. There are 14 busbars, 35 branches and 41 lines. The equivalent $\Pi$ model is used to represent each transmission line. The system consists of 10 loads connected at busbars 3-5 and 8-14 that contains two harmonic sources. One is a twelvepulse HVDC terminal at bus 3 and the other is a SVC at bus 8 . Because the system has balanced bus loads and the transmission lines are transposed, a balanced harmonic analysis is generally sufficient for determining harmonic distortion levels in this case ${ }^{[15]}$. There are 69 possible measurement locations (m), given that there are 14 injections current measurements, 14 busbars voltage measurements and 41 lines current measurements (both sending and receiving ends).

In order to obtain a unique solution for harmonic state estimation, the minimum required numbers of harmonic instruments has to be equal to the number of state variables. As a result, the optimal number of

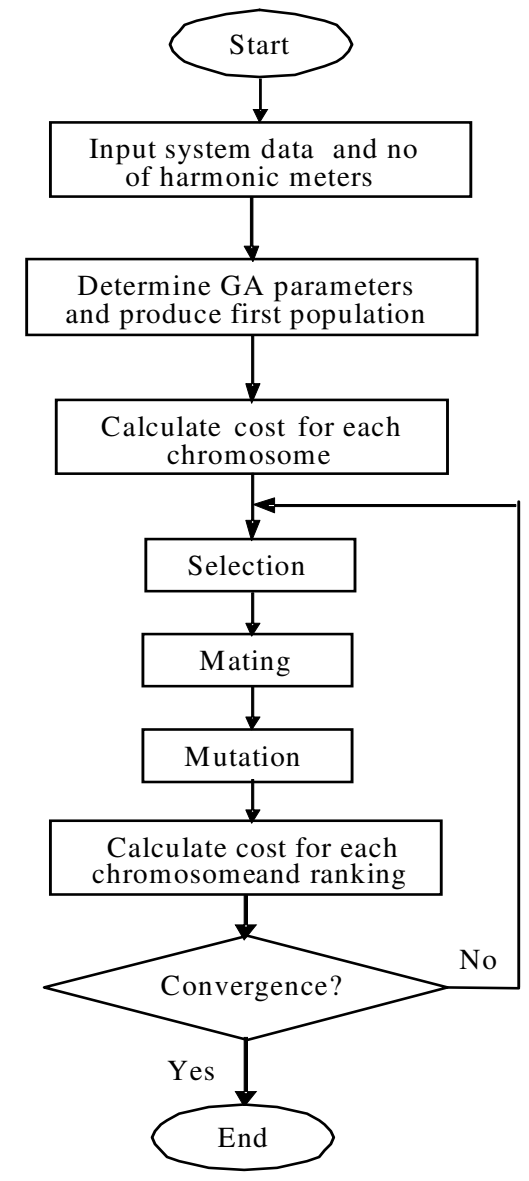

Fig. 1: Flowchart of optimal measurement placement algorithm

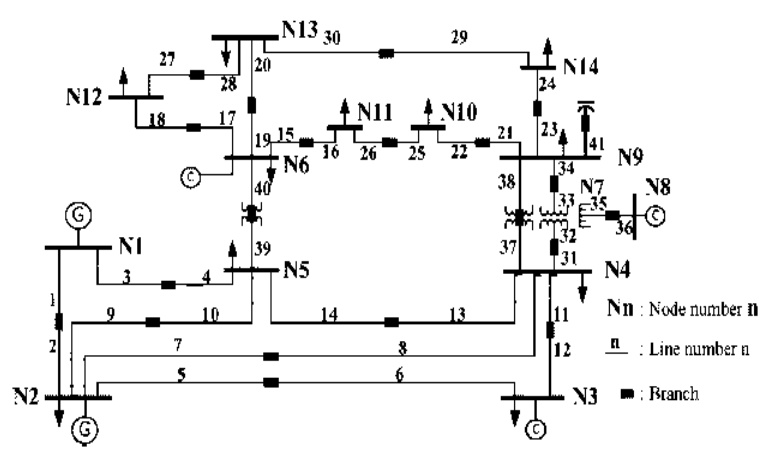

Fig. 2: IEEE 14-bus test system

harmonic instruments is equal to the number of state variables. Actually the state variable of the test system is 14, which using HSE algorithm can be reduced to the number of suspicious nodes (i.e. 10).

The proposed algorithm for measurement placement is written using MATLAB ${ }^{[17]}$. 
Am. J. Applied Sci., 5 (11): 1499-1505, 2008

Table 1: Measurement placement: without virtual measurement

\begin{tabular}{lll}
\hline Proposed criteria & CN criteria & Measuring locations \\
\hline 8 & - & Injection currents \\
$1,3,6,7,9-11,13,14$ & $3,8-14$ & Bus voltages \\
- & 7,40 & Line currents \\
2.8 & 1.6 & CN \\
10 & 8 & NOV \\
0.05 & 0.08 & Error $(\%)$ \\
\hline
\end{tabular}

Table 2: Measurement placement: with virtual measurement

\begin{tabular}{lll}
\hline Proposed criteria & CN criteria & Measuring locations \\
\hline $8,(1,2,6,7)$ & $(1,2,6,7)$ & Injection currents \\
$1,3,6,7,9-11,13,14$ & $3,8-14$ & Bus voltages \\
- & 7,40 & Line currents \\
39.8 & 23.1 & CN \\
14 & 8 & NOV \\
0.00 & 0.06 & Error $(\%)$ \\
\hline
\end{tabular}

Investigating of the proposed criteria: In this part, the performance of the proposed criteria in (9) is compared with the minimum condition number criteria. Also, the sequential procedure has been used for harmonic meter placement.

Furthermore the measurement placements are different among harmonic orders, but all of the measurement placements from all harmonic orders are sufficient to uniquely calculate all state variables for all harmonic orders of the system correctly.

The measurement placements of this system, using minimum condition number of the measurement matrix of the 5th harmonic, are node voltages at busbars 3 and 8-14 and line currents in lines 7 and 40.

On the other hand, if proposed criteria in (9) at the 5 th harmonic is used, the measurement placement will be the node voltages at busbars 1, 3, 6, 7, 9-11, 13 and 14 and injection current at busbar 8, resulting in the fully observable system.

Table 1 shows the results for two different criterions that verify the effectiveness of the proposed criteria.

Table 2 shows the results where the virtual measurements (harmonic current injections at nonharmonic source busbars) have been included. The numbers in parenthesis at Table 2 are the locations related to the virtual measurements.

According to Table 1 and 2, the following results are obtained:

- Although the $\mathrm{CN}$ of measurement matrix that obtained based on the proposed criteria is more compared to the $\mathrm{CN}$ of measurement matrix of the minimum condition number criteria, but as the NOV has been increased and the square of errors between true and estimated fifth harmonic voltages has been decreased, thus these locations are more desirable for meters
Table 3: Measurement Placement: Using Minimum CN Criteria

\begin{tabular}{llll}
\hline Measuring locations & CE method & SP method & BGA method \\
\hline Injection currents & - & - & - \\
Bus voltages & $3,8-14$ & $3,8-14$ & $3,8-14$ \\
Line currents & 6,31 & 7,40 & 6,31 \\
NOV & 9 & 8 & 9 \\
Error (\%) & 0.05 & 0.08 & 0.05 \\
Time (min) & 235 & 7 & 19 \\
\hline
\end{tabular}

Table 4: Measurement Placement: Using the Proposed Criteria

\begin{tabular}{llll}
\hline Measuring locations & CE method & Sp method & BGA method \\
\hline Injection currents & 6,8 & 8 & 6,8 \\
Bus voltages & $1,3,7,10,11$, & $1,3,9-11,6$, & $1,3,7,10$, \\
& 13,14 & $7,13,14$ & $11,13,14$ \\
Line currents & 31 & - & 31 \\
NOV & 10 & 10 & 10 \\
Error (\%) & 0.035 & 0.05 & 0.035 \\
Time (min) & 251 & 12 & 25 \\
\hline
\end{tabular}

- By using virtual measurements, the error of estimation is decreased. Thus to improve the quality of estimation, these can be included in the measurement matrix

- By adding the virtual measurements to the obtained combination for meters based on minimum condition number criteria, the NOV has not been increased and only the error of state estimation has been decreased a little. Whereas by adding them to the obtained combination for meters based on proposed criteria, the system has been completely observable and the error of state estimation approaches to zero

Investigating of the proposed BGA: In this section, to test the effectiveness of the proposed BGA for optimal harmonic meter placement; simulation studies have been carried out in IEEE 14-bus test system. The results obtained by the proposed BGA have been compared with those obtained by both $\mathrm{CE}$ and sequential techniques. In Table 3 and 4 the results have been shown based on minimum $\mathrm{CN}$ of the measurement matrix and proposed criteria, respectively. It's noted that in these Tables the virtual measurements have not been included.

Based on Table 3 and 4, it is observed that BGA is able to find out the optimal combination of meters with significantly less computational time compared to the $\mathrm{CE}$ technique, whereas, the sequential procedure fails to do so.

\section{CONCLUSIONS}

In this research a new method based on SVD and BGA has been proposed to place meters optimally for estimating and identifying the unknown harmonic sources. The performance of the proposed criteria has 
been compared with the minimum condition number criteria and performance of the proposed optimization technique (BGA) has been compared with those of $\mathrm{CE}$ and sequential techniques in IEEE 14-bus test system. Based on this study the major conclusions of this work are:

- Minimizing the $\mathrm{CN}$ of the measurement matrix doesn't necessarily lead to the better estimation quality

- Although the $\mathrm{CN}$ of the obtained meters combination based on proposed criteria is not less as compared to the obtained meters combination based on minimum condition number criteria; but as the measuring residual errors has been minimized and also the NOV increased, these locations are more desirable

- The proposed BGA-based method finds the optimal meter locations while the sequential technique can guarantee only near optimal solutions. Moreover, BGA is capable of finding out the optimum combination in significantly less computational time as compared to the $\mathrm{CE}$ technique

- To improve the quality of harmonic state estimation, Virtual measurements can be included in the measurement matrix

\section{REFRENCES}

1. Arrillaga, J., D. Bradley and D. Bodger, 2003. Power System Harmonics. John Wiley and Sons, New York.

2. 1992. IEEE recommended practice and requirements for harmonic control in electric power system. IEEE Std. 519.

3. Heydt, G.T., 1989. Identification of harmonic sources by a state estimation technique. IEEE Trans. Power Delivery, 4: 569-576.

4. Farach, J.E., W.M. Grady and A. Arapostathis, 1993. An optimal procedure for placing sensors and estimating the locations of harmonic sources in power system. IEEE Trans. Power Delivery, 8 (3): 1303-1310.

5. Watson, N.R., J. Arrillaga and Z.P. Du, 2000. Modified symbolic observability for harmonic state estimation. IEE Proc. Generation, Transmission and Distribution, 147 (2): 105-111.
6. Chakphed, M. and N.R. Watson, 2005. An optimal measurement placement method for power system harmonic state estimation. IEEE Trans. Power Delivery, 20 (2): 1514-1521.

7. Ashwani, K. and D. Biswarup, 2005. Genetic algorithm-based meter placement for static estimation of harmonic sources. IEEE Trans. Power Delivery, 20 (2): 1088-1096.

8. Matair, S.S., N.R. Watson, K.P. Wong, V.L. Pham and J. Arrillaga, 2000. Harmonic state estimation: A method for remote harmonic assessment in a deregulated utility network. Proc. Int. Conf. Electric Utility Deregulation, Restructuring, Power Technologies, 41-46.

9. Du, Z.P., J. Arrillaga and N.R. Watson, 1996. Continuous harmonic state estimation of power systems. Proc. Inst. Elec. Eng., Gen., Transm. Distrib., 329-336.

10. Meliopoulos, A.P.S., F. Zhang and S. Zelingher, 1994. Power system harmonic state estimation. IEEE Trans. Power Delivery, 9: 1701-1709.

11. Arrillaga, J., N.R. Watson and S. Chen, 2000. Power System Quality Assessment. John Wiley and Sons, New York.

12. Holten, L., A. Gjelsvik, S. Aam, F.F. Wu and W.H.E. Liu, 1998. Comparison of different methods for state estimation. IEEE Trans. Power Syst., 3: 1798-1806.

13. Thukaram, D., J. Jerome and C. Surapong, 2000. A robust three-phase state estimation algorithm for distribution networks. Elect. Power Syst. Res., 55: 191-200.

14. Matharad, C., S. Premrudeepreechacharn and N.R. Watson, 2003. Power system state estimation using singular value decomposition. Elect. Power Syst. Res., 67: 99-107.

15. Task Force on Harmonics Modeling and Simulation Transmission and Distribution Committee, 1999. Task force for harmonic modeling and simulation. IEEE Trans. Power Delivery, 14: 579-587.

16. Haupt, R.L. and S.E. Haupt, 2003. Practical Genetic Algorithms. John Wiley and Sons, New York.

17. MATLAB Software (Version 7.2), Mathwork Inc. 